\title{
Assessment of Postoperative Pain in Nursing
}

\author{
Iveta Strode*, Inara Logina** \\ *Pauls Stradins Health and Social Care College, Riga, Latvia \\ **Riga Stradins University, Department of Neurology, Latvia
}

\begin{abstract}
Summary
Introduction. Postoperative pain is a typical form of acute pain and is connected with extensive traumatization of tissue and operation wound traumatic edema, which in its turn, becomes a source of permanent nociceptive impulses. Insufficiently controlled postoperative pain is a factor of risk for the development of various pathologies, postoperative complications, as well as chronic pain.
\end{abstract}

Aim of the study. To study the usage of postoperative period pain intensity and quality assessment scales in the clinical practice of nursing, as well as availability of methods.

Materials and methods. Inquiry utilized quantitative research method - questionnaire. The questionnaire embraced 263 patients and 309 nurses, working in surgical profile. Survey was carried out in surgical profile wards in Riga and regional clinics of Latvia.

Results. Prevalent method, in our investigation of pain intensity evaluation, is patient's oral subjective and objective assessment of condition. Such method is mentioned by $87 \%$ (269/309) of respondents. A conventional and acknowledged method of pain objectivization - verbal descriptor pain intensity scale is applied by $22 \%(69 / 309)$ respondents, but visual analogue scale is utilized only by $5 \%$ (15/309) respondents. Assessment of postoperative pains as systematic and planed operation was marked by $41 \%$ $(126 / 309)$ of surgical nurses.

Conclusions. Prevailing method in the clinical practice of pain assessment is patient's subjective and objective evaluation of condition. However, this method is insufficiently recorded and objectivizated. Therefore, it is an actual problem in surgical patient care and shows necessity for standards and improvement of postoperative pain management.

Key words: acute pain, assessment of pain, assessment of psychological factors, nursing, pain management, postoperative pain in adults, postoperative patient care.

\section{INTRODUCTION}

International Association for the Study of Pain defines pain as an unpleasant sensations and emotions related to real or potentially possible damage of tissue or is described as such damage (1). Despite its various mechanisms of origin and causes, pain always is a subjective feeling with multidimensional nature, formed by physical, emotional and cognitive components.

Postoperative pain is a typical form of acute pain and is connected with extensive traumatization of tissue and operation wound traumatic edema, which in its turn, becomes a source of permanent nociceptive impulses. Insufficiently controlled postoperative pain is a factor of risk for the development of various pathologies, postoperative complications, as well as chronic pain. Pain causes response from the cardiovascular system. It results in disturbances of the heart rhythm, tachycardia, hypertension, disturbance of microcirculation, venous obstruction. Pain gives unfavorable effect on other body systems and can trigger different complications. Therefore, care should ensure the complete control over rational influence and maximal decreasing of pain.

The aim of the patients with acute pain is maximal reduction of pain, lesser effective dose of medication, maximal level of activity, improvement of psychological effects, utilizing continuity principle in objective assessment of pain.

Scales and questionnaires, based on critical self-analyses and self-assessment of pain from an individual's subjective feelings, are used in the clinical practice in order to assess intensity and quality of pain. Simple one-dimensional pain assessment scales are a visual analogue scale, a numerical analogue scale and a verbal pain intensity scale (4). Utilization of the pain assessment scales in patient care process is a prerequisite for a purposeful care.

Professional competence of a nurse is characterized by her ability to solve the health care problems of a patient, analyzing her own experience and taking advantage of theoretical knowledge. Two sisters - scientists Patricia Benner and Judith Vrubel in professional competence theory describe skills and levels of nurse knowledge. They update the levels of competence - from trainee to expert. This competence is complicated and bases on scientific studies and theoretical acknowledgement. Benner defines competence as "comprehension of definite area, performance of skills in this area as well as identification of this area, understanding and utilization of aims and idea" (8).

In pain care nurse is an integral and essential member of team, working together with an anesthetist, surgeon and other nursing personnel.

\section{AIM OF THE STUDY}

To study the usage of postoperative period pain intensity and quality assessment scales in the clinical practice of nursing, as well as availability of methods. 


\section{MATERIALS AND METHODS}

Survey utilized quantitative research method. As an investigation tool was chosen questionnaire. Inquiry forms for surgical profile patients and nursing staff were created during the study. Survey was carried out in surgical profile wards in Riga and regional clinics of Latvia. Questionnaire embraced 263 patients and 309 nurses, working in surgical profile.

Questions of the questionnaire for surgical profile nursing staff were subdivided in the following groups:

- general part (age, gender, professional education, professional work experience);

- questions on utilization of postoperative pain assessment methods and its efficiency in the daily nursing process;

- questions characterizing model of postoperative pain assessment organization and the factors influencing usability of postoperative pain assessment methods.

Questions of the questionnaire for surgical profile patients were subdivided in the following groups:

- general part (age, gender, education, occupation);

- questions on the utilization of pain assessment methods in postoperative period;

- questions characterizing subjective feelings and factors in postoperative period, influencing patient's perception of pain.

\section{RESULTS}

Demographic analysis of 309 nurses - respondents: 18\% of them are 21-30 years old, $28 \%-31-40$ years old, $33 \%-41-50$ years old, $13 \%-51-60$ years old, $8 \%$ is older than 60 years. More than $50 \%$ of respondents have length of service in profession $20-25$ years, $66 \%$ have work experience in surgical profile patient nursing more than six years. $53 \%$ of respondents have secondary vocational education, $18 \%$ - the first level higher vocational education and $29 \%$ have higher education. $98 \%$ of respondents were women, $2 \%-$ men.

Demographic analysis of 263 patients- respondents: 156 women and 107 men. $62 \%$ (163/263) of them have secondary or secondary vocational education, $29 \%$ (76/263) - higher education and only 9\% (24/263) primary school education. Most of them - 36\% (95/263) undergo treatment in abdominal surgery, $11 \%(29 / 263)$ in blood vessel surgery, others in neurosurgery, cardiosurgery, traumatology and other wards 69\% $(181 / 263)$ patients had planned operations.

Characterization of Pain Assessment Methods

In clinical wards, included in our study, dominant pain assessment method is patient's subjective and objective oral evaluation of situation (Fig.1).

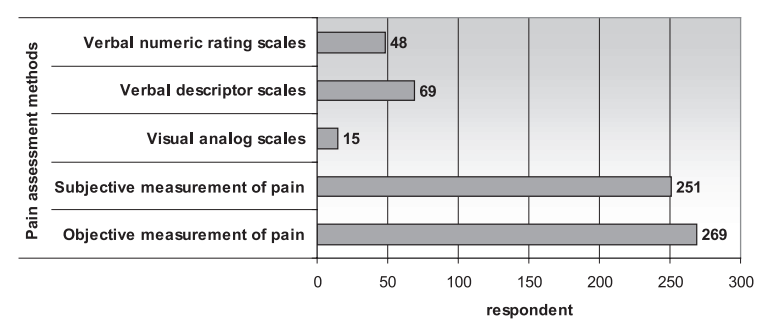

Fig. 1. Application of postoperative pain assessment methods according to the results of nurse questionnaire

Analyzing frequency of pain assessment application, the visual analogue scale in everyday postoperative patient care use only 3\% (9/309) respondents, the verbal descriptor scale $-8 \%(24 / 309)$ and the verbal numeric rating scale $-6 \%(18 / 309)$.

Point of view of nurses- respondents is that assessment of pain is area of responsibility of nurse and one of components of nursing, whereas from the point of view of patients only 236 respondents is of same opinion.

Performing statistical credibility evaluation on frequency of usability of assessment of the subjective condition method between the nurses with secondary vocational and higher education (t- 4.386, degree of freedom $238, \mathrm{p}<0.00)$, conclusion is that the nurses with higher education use this method more often. Similar coherence is seen between the nurses with the first level higher education and higher education $(\mathrm{t}-4.093$, degree of freedom 142, p < 0.00), indicating that nurses with the first level higher education use this method more often then the nurses with higher education.

Evaluating usability of the visual analogue scale between the nurses with secondary vocational education and the nurses with the first level vocational higher education $(\mathrm{t}-$ 3.79 , degree of freedom 204, p < 0.00), one can observe that this method is more frequently used by the nurses with the first level vocational higher education.

Usage of the verbal descriptor scale shows that the nurses with the first level vocational higher education prefer this method more often than the nurses with secondary vocational education $(t-2.87$, degree of freedom 204, $p$ $<0.00$ ) and similar coherence is seen between the nurses with secondary vocational education and the nurses with higher education ( $\mathrm{t}-2.431$, degree of freedom $238, \mathrm{p}<0.01)$. The nurses with higher education prefer this method more often than nurses with secondary vocational education.

Frequency of the verbal numeric rating scale usability among the nurses with secondary vocational education and the nurses with the first level vocational higher education $(t-3.79$, degree of freedom 204, $p<0.00)$ shows that the nurses with the first level vocational higher education use this method more often than the nurses with secondary vocational education. Resembling connectedness is recognized among the nurses with secondary vocational education and the nurses with higher education $(t-3.399$, degree of freedom $138, p<$ 
0.00), where the nurses with higher education employ this method more often than the nurses with secondary vocational education.

Medical Therapy in Postoperative Period

$237(n=263)$ respondents answer that in postoperative period they regularly receive painkillers, whereas $55(n=263)$ respondents mention additional medical therapy for reduction of pain.

In the first day after operation 32\% (84/263) of respondents feel moderate pain (4-6 points), 38\% (101/263) - severe aches (7 and more points) and 30\% $(78 / 263)$ of respondents have only mild pain (Fig.2.).

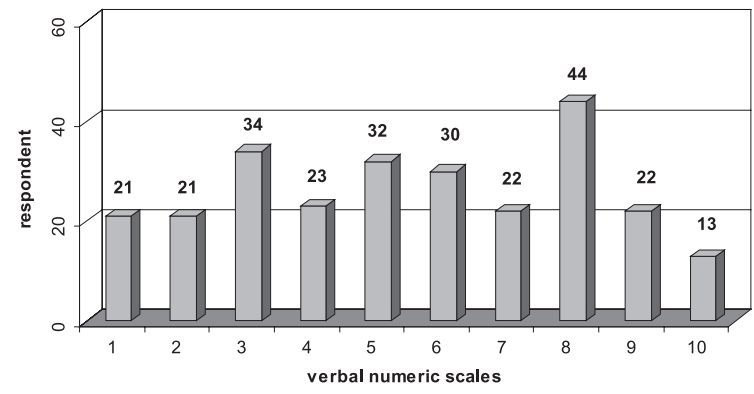

Fig. 2. Postoperarative pain assessment is based upon verbal numeric scales of the first day after surgery

Assessment of postoperative pain as methodical and systematic activity was mentioned by 126 nurses working in surgical profile and 47 respondents consider it as documenting action. Others emphasize that assessment is chaotic, unscheduled and unrecorded action. It is necessary to ensure regular pain assessment organization taking into account also side effects in order to successfully determine appropriate pain management.

\section{DISCUSSION}

Several independent surveys on pain management in postoperative period are carried in different countries. They analyze efficiency of both, medical measures and measures without medication in the reduction of pain syndrome. In spite of the development of pain management in postoperative period, frequency of postoperative pain increases. Inquiry shows that $75 \%$ of patients in postoperative period complain on moderate postoperative pain. Therefore, in clinical nursing the problem of pain is urgent. For example, in the study of postoperative pain in out-department patients, $40 \%$ of the respondents feel moderate pain and $25 \%$ consult medical professionals on the methods of pain reduction (3). In survey on postoperative pain monitoring in early postoperative period, conducted in Germany (110 respondents), were noticed that in the first day after operation intensity of pain is not adequately observed pain is not assessed accordingly or the moderate pain is overestimated. There are many independent studies on postoperative pain in the research of nursing (2).

Nurse - professor J.Travelby (USA) accents that nursing is an interpersonal process in which nurse helps individual, family or society to delay or overcome experience related to illness or suffering (5).

Nursing is a systematic method for determination and problem solving with the planned actions in order to ensure for patient individual care in different health conditions. However, in this inquiry assessment of pain as a regular action mention only 126 respondents (nurses).

One of the tasks in perioperative period is to give a patient information how to assess postoperative pain and possibilities of pain reduction therapies. Management model of anaesthetics is closely connected with the action plan of a particular clinic (6). Efficiency of analgezia, quality of seizure prevention and potency length of medicine should be evaluated. It is important to clarify the time, when analgetic was last time injected and the length of time till pain recrudesce in order to evaluate the signs of tolerance. The first sign of tolerance is decrease of anaesthetic potency length. It is important that assessment of pain should be considered as the fifth vital indicator in nursing. Study of Danish nurses on pain monitoring, conducted in five clinics, conclude that $83,6 \%$ nurses postoperative pain assessment consider as a routine. Whereas $78,1 \%$ of nurses assessment of pain put forward as a vital sign in postoperative period. This inquiry stresses that documentary assessment of pain plays a great role in postoperative pain management (7).

One of essential tasks in postoperative period is to release a patient from distress caused by pain in order to provide comfort for a patient. This is a motivation for humane and ethical nurse practice ensuring to relieve pain for postoperative patient. Still in Latvia there are a few studies on acute pain influence on recovery, progress of disease and outcome as well as assessment of pain intensity and quality.

\section{CONCLUSIONS}

Prevailing method in the clinical practice of pain assessment is patient's subjective and objective evaluation of condition. However, this method is insufficiently recorded and objectivizated. Therefore, it is an actual problem in the surgical patient care and shows necessity for standards and improvement of postoperative pain management.

\section{ACKNOWLEDGEMENT}

Article was created with support of European Social Fund.

\section{Conflict of interest: None}

\section{REFERENCES}

1. Classification of chronic pain. 2nd edition // IASP Press, 1994

2. Gross T., Pretto M., Aeschbach A. Pain management in surgical wards. Quality and solutions for 
improvement in the early postoperative period // Chirurg, 2002; 73:818-826

3. Karanikolas M., Swarm R.A. Perioperative medicine: current trends in perioperative pain management // Anesthesiology Clinics of North America, 2000; 18:579 - 599

4. Le Bel A.A. Assessment of pain // In: Ballantyne J.C. The Massachusetts general hospital handbook of pain management. Third Edition. Philadelphia: Lippincott Williams \& Wilkins; 2006; $58-75$

5. Meleis A.I. Theoretical nursing: Development\& Progress. Philadelphia: J.B. Lippincott Company; $1985 ; 254-263$

6. Odom J. Postoperative patient care and pain management // In: Rothrock J.C. Care of the Patient in Surgery. Printed in the United States of America: Mosby; 2003; 253 - 280

7. Rond M., Wit R., Dam F. The implementation of a pain monitoring programme for nurses in daily clinical practice: results of a follow-up study in five hospitals // Journal of Advanced Nursing, 2001; 35:590_ 598

8. Šilina M., Dupure I. Pacientu izglītošana - māsas kompetence. Rīga: Nacionālais apgāds; 2004; 104

\section{Address:}

Iveta Strode

Pauls Stradins Health and Social Care College

Vidus prospects $36 / 38$,

Jurmala, Latvia, LV-2010

E-mail: ivetastrode@inbox.lv 